\title{
Vortices around Janus droplets under externally applied electrical field
}

\author{
Mengqi Li and Dongqing Li* \\ Department of Mechanical and Mechatronics Engineering, University of Waterloo, \\ Waterloo, Ontario, Canada N2L 3G1
}

*Corresponding author, Address: 200 University Ave. West, Waterloo, Ontario, N2L 3G1 Email: dongqing@uwaterloo.ca (D. Li)

\section{Statement}

The final publication is available at Springer via http://dx.doi.org/10.1007/s10404-016-1741-2. 


\begin{abstract}
In this study, the Janus droplet is an oil droplet covered with aluminum oxide nanoparticles on one side of the droplet surface under applied DC electrical field. The vortices around Janus droplets fixed on a horizontal surface were studied in this paper. A numerical model was set up to simulate the vortices around the Janus droplet in electric field. The simulation results illustrate that the electric field determines the strength of the vortices around a fixed Janus droplet and the surface coverage of the positively charged nanoparticles on a Janus droplet affects the size and location of the vortices. The numerically predicted results were further validated experimentally by visualizing the vortices around Janus droplets in an externally applied DC electric field. Furthermore, as the Janus droplets are generated in electric field, the surface coverage by the nanoparticles depends on the strength of the electric field; therefore, the effect of the electric field on the nanoparticle covered surface area of a Janus droplet and the vortices was analyzed.
\end{abstract}

Key Words: Janus droplet, charged nanoparticles, electro-osmotic flow, vortices 


\section{Introduction}

For a polarizable sphere immersed in an electrolyte solution, when a DC electrical field is applied, electric charges will be induced along the surface of the sphere. The induced charges have opposite signs on the two hemispheres: the hemisphere facing the applied electric field is negatively charged; the other hemisphere is positively charged. Consequently, the induced electric double layer field around the sphere is dipolar; the electroosmotic flows from the two sides of the sphere are in the opposite directions. This will result in vortices around the sphere. Induced charge electroosmotic flows around ideally polarizable objects such as a metal cylinder or sphere have been studied extensively (Levich 1962; Dukhin and Murtsovkin 1986; Dukhin 1986; Bazant and Squires 2004; Yariv 2005; Saintillan et al. 2006a; Saintillan et al. 2006b; Yossifon et al. 2007; Gangwal et al. 2008; Wu et al. 2009; Wu and Li 2009; Bazant and Squires 2010). On the theoretical side, an analytical formulation which can be used to calculate the induced zeta potential along the surface of a 2D metal cylinder of circular cross-section was derived (Bazant and Squires 2004). Wu and Li (2008a) presented a numerical method of evaluating the induced zeta potential on an arbitrary shaped surface of a fully polarizable object. Among experimental studies, Daghighi et al. (2013) investigated the induced charge electroosmotic flow field surrounding a carbon-steel sphere in water and used fluorescent particles to show the flow patterns. Under externally applied DC electric field, four vortices were observed in the vicinity of the metal sphere; the size and the strength of the vortices increase with the electric field. Peng et al. (2014) experimentally studied the induced charge electroosmotic flow around an Au sphere under AC electric field. It was demonstrated that four vortices can also be generated around the Au sphere in AC electric field. The induced vortices around conducting surfaces can be used for many applications: mixing and controlling flow regulating inside microchannels (Wu and Li 2008a)(Wu and Li 2008b)(Zhang et al. 2011), pumping liquid(Kim et al. 2011), separating particles(Zhang and Li 2014) and trapping cells(Ren et al. 2015).

The induced charge electroosmotic flow and vortices were also studied for Janus particles consisting of two halves: one is an electrically conducting metal (i.e., fully polarizable material), the other is electrically non-conducting material (i.e., dielectric). Daghighi and Li (2011) numerically studied the electrical induced vortices around Janus particles. Under DC electric 
field, the induced charges on the metal side of the Janus particle generate two major vortices. They also analyzed the influence of the vortices on the motion of Janus particles in microchannel, and it was found that the vortices may act as the "vortex engine" for the particle's motion. This finding was verified experimentally by measuring the velocities of Janus particles and homogeneous dielectric particles with the same size in externally applied DC electric field (Daghighi et al. 2013). Later on, Peng et al. (2014) experimentally studied the induced vortices around a Janus $\mathrm{Au}-\mathrm{SiO}_{2}$ particle under $\mathrm{AC}$ electric field. Their experimental results indicated that the vortices can always be generated no matter the electric field was parallel or perpendicular to the interface of the two hemispheres of the Janus particle.

For a Janus droplet composed of a negatively charged surface on one side and a positively charged surface on the other side, vortices around the Janus droplet may be expected, similar to a solid Janus particle, under applied electric field. The vortices around Janus droplets have many potential applications. Mixing inside microchannels is a challenge due to small Reynolds number, and the vortices around Janus droplets can enhance the mixing and the Janus droplets may be regarded as micromixers. Furthermore, vortices around Janus droplets may act as "vortex engine", which will affect the motion of Janus droplets. Therefore, the vortices around Janus droplets can be utilized to control the motion and separation of Janus droplets based on surface coverage of different charges and size. However, no paper has been published to report any studies of the electroosmotic flow and the vortices around Janus droplets. One of the reasons is that it is difficult to produce a Janus droplet with opposite signs of surface charges on the two sides. Currently, two main techniques have been developed to generate Janus droplets, the onestep high energy mixing method (Torza and Mason 1970; Hasinovic et al. 2011; Hasinovic and Friberg 2011; Friberg et al. 2013; Ge et al. 2014; Zarzar et al. 2015) and the microfluidic method (Utada et al. 2005; Nie et al. 2006; Pannacci et al. 2008; Guzowski et al. 2012). Janus droplets formed with high energy mixing method are composed of two immiscible oils. As the oil-water interfaces generally carry negative charges, the Janus droplet generated by this method cannot have one side with positive surface charges and the other side with negative surface charges. In the microfluidic method, the Janus droplets are generated inside microchannels. The properties of the two parts of the Janus droplet depend on that of the two immiscible liquid monomers. Generally, the monomers are organic solutions, their interfaces with an aqueous solution all carry 
negative charges; therefore, this method is also not suitable for generating Janus droplets with a positively charged hemisphere and a negatively charged hemisphere.

In this paper, a new method for generating Janus droplets with one positively charged side and one negatively charged side is introduced. Such Janus droplet is formed by covering one side of an oil droplet with positively charged aluminum oxide nanoparticle in an externally applied electric field. The factors that affected the vortices around Janus droplets were studied. A numerical model was built, and the simulations were conducted to investigate the electroosmotic flow and vortices around a Janus droplet. The vortices were studied experimentally and the experimental observation was compared with the numerical simulation results.

\section{Experiments}

The purpose of the experiments was to study the electro-osmotic flow and vortices around Janus droplets. The Janus droplets used in this study were generated by partially covering oil droplets with positively charged aluminum oxide nanoparticles under externally applied electric field. Furthermore, to avoid the unwanted effect of the droplet motion, we conducted the experiments with sessile Janus droplets which were anchored on a solid surface in the aqueous solution, as illustrated in Figure 1.

\subsection{Janus droplet preparation}

To generate a sessile Janus droplet partially covered with aluminum oxide nanoparticles, the aluminum oxide nanoparticle suspension was prepared first. The aluminum oxide nanoparticles of $5 \mathrm{~nm}$ in diameter (US Research Nanomaterials, Inc., Houston, TX, USA) were dispersed into deionized water by using ultrasonic wave: a) Add nanoparticles into deionized water in a beaker; b) Place the beaker into an ultrasonic cleaner for a period of time (approximately 8 minutes) and the nanoparticles can be dispersed under ultrasonic wave. The concentration of the aluminum oxide nanoparticle suspension used in this study is $50 \mathrm{mg} / \mathrm{mL}$.

The sessile oil droplet immersed in water can be generated by following these procedures: a) Pour deionized filtered water into a plastic petri dish; b) Place a piece of cover glass on the surface of water, which wouldn't sink due to the surface tension of water; c) Deposit certain volume of the mineral oil (Sigma-Aldrich, Canada Ltd.) with the viscosity of around $15 \mathrm{cSt}$ at $40^{\circ} \mathrm{C}$ 
on the floating cover glass slide; d) Push the glass slide into water, sitting at the bottom of the petri dish, and a sessile oil droplet attaching on the glass slide formed.

After the sessile oil droplet was generated in water, a certain amount of aluminum oxide nanoparticle suspension prepared before was deposited onto the oil droplet with a digital micropipette. As the aluminum oxide nanoparticles and the oil-water interface carried opposite signs of charges, the particles adhered on the oil droplet and distributed uniformly. If an external DC electric field is applied to the droplet through water at this time, the positively charged nanoparticles at the oil-water interface will be forced to accumulate to one side of the oil droplet and leave a pure oil-water interface on the other side without the presence of nanoparticles. A Janus droplet is formed in this way.

\subsection{Experimental setup}

In order to visualize the fluid flow around the sessile Janus droplet, $1 \mu$ spherical polystyrene particles (Bangs Laboratories Inc., IN, USA) were used as the tracing particles. The experimental system are shown in Figure 2: a petri dish to hold a sessile oil droplet covered with aluminum oxide nanoparticles in water, a DC power supply (CSI12001X, Circuit Specialist Inc., USA), a microscope imaging system (Ti-E, Nikon, Japan). During the experiment, the petri dish was put on the stage of the microscope. The electric field was applied by the DC power supply through two electrodes immersed in the aqueous solution. The strength of the electric field can be adjusted by controlling the output voltage of the DC power supply. The microscope with a digital camera (DS-Qi1Mc, Nikon) was taken to visualize the trace particles' motion around the Janus droplet. The images can be captured by the digital camera and sent to a computer to be saved and analyzed.

\section{Theoretical Model and Numerical Simulation}

Consider an oil Janus droplet, one side with positive surface charges, the other side with negative surface charges, immersed in an infinitely large aqueous solution, electroosmotic flow will be generated around the droplet under electric field. Due to the opposite signs of zeta potentials on different sides of the Janus droplet, vortices will be produced in vicinity of the droplet because of the opposite directions of electroosmotic flow on the two sides of the Janus droplet. A multi- 
physics model is employed to simulate the electric field and flow field of the system, as described in the following sections.

Furthermore, it should be noted that the electroosmotic flow at the liquid-liquid interface has been studied in some papers and the electric double layer plus surface charges (EDL+SC) model was derived to estimate the electroosmotic flow at the liquid-liquid interface (Gao et al. 2005; Lee et al. 2006; Lee and Li 2006; Movahed et al. 2012). The electroosmotic flow near the charged oil-water interface can be described with this model, and the results indicate that the electro-osmotic velocity of water near the interface is similar to that on solid surface if the zeta potentials of the interface and solid surface are the same. In this study, we focused on the electroosmosis of water around sessile Janus droplets; therefore, to simplify the model, the motion inside droplet is neglected and the Janus droplets are regarded as solid particles.

\subsection{Electric field}

After applying the electric field to the system as shown in Figure 2, the electrical potential $\phi_{e}$ in the liquid can be determined by solving the Laplace's equation:

$$
\nabla^{2} \phi_{e}=0
$$

The corresponding boundary conditions are:

$$
\begin{aligned}
& \vec{n} \cdot \nabla \phi_{e}=0 \text { at the oil droplet surface } \\
& \phi_{e}=\phi_{0} \text { at the electric field inlet } \\
& \phi_{e}=0 \text { at the electric field outlet }
\end{aligned}
$$

where $\vec{n}$ is the unit vector normal to the boundary.

\subsection{Flow field}

The Navier-Stokes equation

$$
\rho\left[\frac{\partial \vec{u}}{\partial t}+\vec{u} \cdot \nabla \vec{u}\right]=-\nabla P+\mu \nabla^{2} \vec{u}
$$

and the continuity equation

$$
\nabla \cdot \vec{u}=0
$$


are the governing equations of the incompressible liquid flow around the droplet.

Here, $\mu$ is the viscosity of the aqueous solution, $\rho$ is the density of the solution, $\vec{u}$ is the velocity vector and $\nabla P$ is the pressure gradient. $\partial \vec{u} / \partial t$ is the transient term, which can be neglected from Eq. (5) by considering the steady-state flow.

The boundary conditions for the flow field are:

$$
\begin{aligned}
& \vec{u}=-\frac{\varepsilon \varepsilon_{0} \zeta_{d}}{\mu} \vec{E} \\
& \vec{n} \cdot \nabla P=0 \text { at the droplet surface } \\
& \vec{n} \cdot \nabla \vec{u}=0 \\
& P=0 \text { at far field boundaries }
\end{aligned}
$$

$\vec{E}=-\nabla \phi_{e}$ is the local applied electric field, which can be obtained by solving the electric field as described by Eq.(1). $\varepsilon_{0}$ and $\varepsilon$ are the dielectric constant of the vacuum and the aqueous solution, respectively. $\zeta_{d}$ is the zeta potential of the Janus droplet, which has different values on different sides of Janus droplets.

\subsection{Numerical simulation}

As mentioned above, electroosmotic flow can be generated around the droplet under electric field. Due to the opposite signs of zeta potential on the opposite sides of the Janus droplet, electroosmotic flows are in opposite directions from different sides of the droplet. Thus, vortices will form in vicinity of the Janus droplet. It is easy to understand that the size, location and strength of the vortices depend on the applied electric field as well as the surface coverage of the Janus droplet by the nanoparticles.

In this study, the effects of the surface coverage of the Janus droplet by the nanoparticles and the externally applied electric field on the vortices were investigated. As shown in Figure 3, a sessile droplet of $d=200 \mu \mathrm{m}$ in diameter is placed in the center of the computation domain. The length and width of the computation domain are $L=W=20 \mathrm{~d}$. The theoretical model shown above was solved with the commercial software COMSOL 4.3b. The total number of unstructured elements used for calculation was 100,494. As this study considers the Janus droplet that is 
generated by covering one side of the oil droplet with positively charged aluminum oxide nanoparticles in externally applied DC field. The zeta potential on the pure oil-water interface $\zeta_{n}$ is set to be $-40 \mathrm{mV}$ (Graciaa et al. 2002), and the zeta potential on the surface of the nanoparticles $\zeta_{p}$ is $+60 \mathrm{mV}$ (Hunter 1981; Rezwan et al. 2004; Timofeeva et al. 2007). Other parameter values used in the simulations are shown in Table 1.

\section{Results and Discussion}

For a Janus droplet with one side carrying positive surface charges and the other side carrying negative surface charges, vortices can be formed in the neighborhood of the Janus droplet under externally applied electrical field. Figure 4(a) shows the typical flow stream patterns around a Janus droplet of $200 \mu \mathrm{m}$ in diameter under applied electric field $40 \mathrm{~V} / \mathrm{cm}$. In this case, the positive surface charges are on the right-hand side of the droplet; and the negative surface charges are on the left-hand side of the droplet. As one can see in this figure, two vortices were generated around the droplet because of the zeta potential difference between the two sides of the droplet. Because the absolute value of zeta potential on the right hemisphere $(+60 \mathrm{mV})$ is larger than that on the left hemisphere $(-40 \mathrm{mV})$, the electroosmotic flow on the right hemisphere dominates the flow and hence the two vortices stay on the left side. Verification experiment was conducted at the same electric field of $40 \mathrm{~V} / \mathrm{cm}$. A Janus droplet with the diameter of $170 \mu \mathrm{m}$ was generated by following the procedures described above. As shown in Figure 4(b), two vortices formed on the left side of the Janus droplet. The comparison of Figures 4(a) and 4(b) indicates that the numerical prediction agrees well with the experimental observation.

\subsection{Effect of the applied electrical field}

It is well known that electroosmotic velocity is directly proportional to the applied electric field. To study how the vortices vary with electrical field, the numerical simulations for three different strengths of electric fields were conducted. Figure 5 shows the flow fields around the same Janus droplet under three different electric fields, $40 \mathrm{~V} / \mathrm{cm}, 50 \mathrm{~V} / \mathrm{cm}$ and $60 \mathrm{~V} / \mathrm{cm}$. It can be seen from Figure 5 that two vortices were formed on the left-hand side (negatively charged surface) of the Janus droplet under different applied electrical fields. Comparison of the three cases in Figure 5 clearly shows that when the surface coverage of the Janus droplet by the nanoparticles is fixed, $50 \%$ in this case, the externally applied electrical field has no appreciable effect on the location 
and size of the vortices. As the electric field increases, the velocity of the two opposite electroosmotic flows from the two hemisphere surfaces all increases. Therefore, the locations of the vortices remain the same. However, the rotation speed of the vortices becomes larger.

In order to experimentally verify the prediction of the above numerical simulations, an oil droplet covered with aluminum oxide nanoparticles was generated in a plastic petri dish. To make the accumulation area of the nanoparticles unchanged under different externally applied electrical fields, the electric field of $60 \mathrm{~V} / \mathrm{cm}$ was applied from left to right for a period of 2 3 minutes. This electrical field was strong enough to push the nanoparticles to accumulate to the right hemisphere of the oil droplet and make them tightly packed with each other; so that, when the external electrical field was reduced to $50 \mathrm{~V} / \mathrm{cm}$ or $40 \mathrm{~V} / \mathrm{cm}$, the aggregated nanoparticles were kept in the original area (the right hemisphere). In this way, a Janus droplet with a fixed surface coverage by the nanoparticles under different applied electric fields can be studied. Figure 6 shows the vortices around the Janus droplet of $170 \mu \mathrm{m}$ in diameter under different applied electrical fields. In order to see the vortices clearly, only the vortices near the up-left quarter of the droplet were shown. All of the three pictures presented in Figure 6 were taken with the same exposure time of $200 \mathrm{~ms}$. Therefore, the strength of the vortices under different applied electric field can be obtained by comparing the length of the streak lines of the tracing particles. By comparing the three pictures in this figure, the same conclusion can be obtained: as the electrical field increases, the strength of the vortices increases while the location and size of the vortices remains unchanged.

\subsection{Effect of the surface coverage of the Janus droplet by the nanoparticles under the same electrical field}

The surface coverage of the Janus droplet by the nanoparticles is another factor that will affect the vortices. As mentioned above, when the nanoparticle surface coverage of the Janus droplet is $50 \%$, because the absolute value of the zeta potential on the surface of aluminum oxide nanoparticles is larger than the zeta potential of the oil-water interface, the electroosmotic flow on this hemisphere covered with nanoparticles will dominate the flow around the droplet, and two vortices will be generated on the other hemisphere of the droplet. When the accumulation area of the nanoparticles is larger, the electroosmotic flow on the positively charged surface becomes dominant over the opposite electroosmotic flow generated on the negatively charged 
surface. This results in vortices on the negatively charged side of the droplet. However, when the accumulation area of the positively charged nanoparticles is small enough, the electroosmotic flow on the negatively charged surface will dominate the flow field around the droplet and the two vortices will be positioned on the positively charged side of the droplet. Figure 7 shows the vortices around Janus droplets with different surface coverage by the positively charged nanoparticles. As shown in Figure 7(a), when the positively charged section (blue area) is larger than the negatively charged section (red area), the electroosmotic flow from the blue area is stronger which forced two vortices to be formed on the other side of the oil droplet. However, the vortices will occur on the positively charged side when the surface coverage by the positively charged nanoparticles becomes sufficiently small and the electroosmotic flow from the negatively charged section becomes dominant, as shown in Figure 7(b).

Figure 8 shows the experimentally observed change of the vortices position with the surface coverage of the droplet by positively charged nanoparticles. The Janus droplets with different surface coverages were generated by depositing different volumes of nanoparticle suspension over the oil droplet. Applying a larger volume of the nanoparticle suspension results in a larger number of nanoparticles on the droplet surface. With the externally applied electric field unchanged, at the final state, the larger number of nanoparticles leads to a larger accumulation area on the droplet surface. As can be seen in Figure $8(\mathrm{a})$, when $\theta \approx 110^{\circ}$, i.e., the positively charged nanoparticles cover the majority of the droplet surface, vortices form on the side of the pure oil-water interface. However, when $\theta \approx 60^{\circ}$, i.e., the positively charged nanoparticles cover

only a small fraction of the droplet surface on the right-hand side, vortices occur on the side of the droplet facing the negative electrode, as shown in Figure 8(b). In order to show the vortices clearly, only one vortex and one quarter of the droplet were shown in Figure 8. Clearly, the predicted results by numerical simulation (Figure 7) and the experimental observation (Figure 8) are in good agreement.

\subsection{Effect of the electrical field on the surface coverage of Janus droplets and vortices}

In section 4.1, to analyze the influence of applied electric field on the vortices, we kept the surface coverage of the Janus droplet unchanged by making the nanoparticles tightly packed with each other under high electric field. However, under relative low electrical field for limited time, 
the nanoparticles and cluster won't aggregate with each other, and the nanoparticle surface coverage of the Janus droplet is dependent on the applied electric field. When the external electrical field is applied, the positively charged nanoparticles move at the interface under the external electrical field force and accumulate to one polar of the oil droplet. As the accumulation of these nanoparticles, the electrostatic repulsive force between the particles gets larger. At final state, the two forces acting on the nanoparticles, external electrical field force and repulsive force, are balanced, and the surface coverage by nanoparticles of the Janus droplet remains unchanged. However, once the strength of the electric field changes, the electrical field force acting on the nanoparticles changes, which will result in the variation of the nanoparticle surface coverage. For example, when the electric field increases, the electrical field force will increase and the distance between the nanoparticles will decrease; therefore, the nanoparticle surface coverage decreases finally.

Figure 9 shows the variation of the surface coverage by nanoparticles and vortices around the same Janus droplet under different electric fields, 40V/cm and 60V/cm. As shown in Figure 9(a), under the electric field $40 \mathrm{~V} / \mathrm{cm}$, the nanoparticles are pushed to accumulate to the right side of the oil droplet and leave the pure oil-water interface on the left side. Small vortex forms on the side of the pure oil-water interface. When the electric field increases to 60V/cm (Figure 9(b)), the accumulation area of the nanoparticles decreases and leave a larger blank area on the other side; therefore, the vortex get larger and stronger.

\section{Conclusion}

Vortices around Janus droplets are studied in this paper. The results of the numerical simulations show that two vortices can be generated in the surrounding area of the Janus droplet under externally applied electrical field. By comparing the numerical results under different circumstances, the following two conclusions can be drawn: 1) If the surface coverage of the Janus droplet by the positively charged nanoparticles is the same, the strength of the vortices increases with the applied electric field; 2) The variation of the surface coverage of the droplet by the positively charged nanoparticles will result in the change of the size and the location of the vortices. These findings are validated experimentally by visualizing the vortices around Janus droplets that are partially covered with positively charged aluminum oxide nanoparticles. 
Furthermore, as this type Janus droplets are generated under electrical field, the strength of the externally applied electric field affects the accumulation area of the particles on the droplet (the surface coverage) within a certain range of the electrical field. Generally, the accumulation area of positively charged particles decreases as the increase of the strength of the electric field. Therefore, the size, position and strength of vortices vary with the electric field, which has been validated through experiment. The findings in this paper are important which can be used to develop novel technology for sorting and separation Janus droplets.

\section{Acknowledgements}

The authors appreciate the financial support of the Natural Sciences and Engineering Research Council (NSERC) of Canada through a research grant to $\mathrm{D}$. Li. 


\section{References}

Bazant MZ, Squires TM (2004) Induced-charge electrokinetic phenomena: theory and microfluidic applications. Phys Rev Lett 92:066101. doi: 10.1103/PhysRevLett.92.066101

Bazant MZ, Squires TM (2010) Induced-charge electrokinetic phenomena. Curr Opin Colloid Interface Sci 15:203-213. doi: 10.1016/j.cocis.2010.01.003

Daghighi Y, Li D (2011) Micro-valve using induced-charge electrokinetic motion of Janus particle. Lab Chip 11:2929-2940. doi: 10.1039/c1lc20229d

Daghighi Y, Sinn I, Kopelman R, Li D (2013) Experimental validation of induced-charge electrokinetic motion of electrically conducting particles. Electrochim Acta 87:270-276. doi: 10.1016/j.electacta.2012.09.021

Dukhin AS (1986) Pair interaction of disperse particles in electric field. 3. Hydrodynamic interaction of ideally polarizable metal particles and dead biological cells.

Dukhin AS, Murtsovkin VA (1986) Pair interaction of particles in electric field. 2. Influence of polarization of double layer of dielectric particles on their hydrodynamic interaction in a stationary electric field.

Friberg SE, Kovach I, Koetz J (2013) Equilibrium topology and partial inversion of janus drops: A numerical analysis. ChemPhysChem 14:3772-3776. doi: 10.1002/cphc.201300635

Gangwal S, Cayre OJ, Bazant MZ, Velev OD (2008) Induced-charge electrophoresis of metallodielectric particles. Phys Rev Lett 100:058302. doi: 10.1103/PhysRevLett.100.058302

Gao Y, Wong TN, Yang C, Ooi KT (2005) Transient two-liquid electroosmotic flow with electric charges at the interface. Colloids Surfaces A Physicochem Eng Asp 266:117-128. doi: $10.1016 /$ j.colsurfa.2005.05.068

Ge L, Shao W, Lu S, Guo R (2014) Droplet topology control of Janus emulsion prepared in onestep high energy mixing. Soft Matter 10:4498-4505. doi: 10.1039/c4sm00456f

Graciaa A, Creux P, Dicharry C, Lachaise J (2002) Measurement of the Zeta Potential of Oil Drops with the Spinning Tube Zetameter. J Dispers Sci Technol 23:301-307. doi: 10.1081/DIS-120003321

Guzowski J, Korczyk PM, Jakiela S, Garstecki P (2012) The structure and stability of multiple micro-droplets. Soft Matter 8:7269-7278. doi: 10.1039/c2sm25838b

Hasinovic H, Friberg SE (2011) One-step inversion process to a Janus emulsion with two mutually insoluble oils. Langmuir 27:6584-6588. doi: 10.1021/la105118h 
Hasinovic H, Friberg SE, Rong G (2011) A one-step process to a Janus emulsion. J Colloid Interface Sci 354:424-426. doi: 10.1016/j.jcis.2010.10.004

Hunter RJ (1981) Zeta Potential in Colloid Science: Principles and Applications. Academic Press, New York

Kim W, Ryu JC, Suh YK, Kang KH (2011) Pumping of dielectric liquids using non-uniformfield induced electrohydrodynamic flow. Appl Phys Lett 99:224102. doi: $10.1063 / 1.3665403$

Lee JSH, Barbulovic-Nad I, Wu Z, et al (2006) Electrokinetic flow in a free surface-guided microchannel. J Appl Phys 99:54905. doi: 10.1063/1.2177428

Lee JSH, Li D (2006) Electroosmotic flow at a liquid-air interface. Microfluid Nanofluidics 2:361-365. doi: 10.1007/s10404-006-0084-9

Levich VG (1962) Physicochemical hydrodynamics: (by) veniamin G. Levich. Transl. by scripta technica, inc. Prentice-Hall

Movahed S, Khani S, Wen JZ, Li D (2012) Electroosmotic flow in a water column surrounded by an immiscible liquid. J Colloid Interface Sci 372:207-211. doi: 10.1016/j.jcis.2012.01.044

Nie Z, Li W, Seo M, et al (2006) Janus and ternary particles generated by microfluidic synthesis: Design, synthesis, and self-assembly. J Am Chem Soc 128:9408-9412. doi: 10.1021/ja060882n

Pannacci N, Bruus H, Bartolo D, et al (2008) Equilibrium and Nonequilibrium States in Microfluidic Double Emulsions. Phys Rev Lett 101:164502. doi: 10.1103/PhysRevLett.101.164502

Peng C, Lazo I, Shiyanovskii S V., Lavrentovich OD (2014) Induced-charge electro-osmosis around metal and Janus spheres in water: Patterns of flow and breaking symmetries. Phys Rev E 90:051002. doi: 10.1103/PhysRevE.90.051002

Ren Y, Liu W, Jia Y, et al (2015) Induced-charge electroosmotic trapping of particles. Lab Chip 15:2181-2191.

Rezwan K, Meier LP, Rezwan M, et al (2004) Bovine serum albumin adsorption onto colloidal Al2O3 particles: a new model based on zeta potential and UV-vis measurements. Langmuir 20:10055-10061. doi: 10.1021/la048459k

Saintillan D, Darve E, Shaqfeh ES (2006a) Hydrodynamic interactions in the induced-charge electrophoresis of colloidal rod dispersions. J Fluid Mech 563:223-259. doi: $10.1017 /$ S0022112006001376 
Saintillan D, Shaqfeh ES, Darve E (2006b) Stabilization of a suspension of sedimenting rods by induced-charge electrophoresis. Phys Fluids 18:121701. doi: 10.1063/1.2404948

Timofeeva E V., Gavrilov AN, McCloskey JM, et al (2007) Thermal conductivity and particle agglomeration in alumina nanofluids: Experiment and theory. Phys Rev E 76:061203. doi: 10.1103/PhysRevE.76.061203

Torza S, Mason SG (1970) Three-phase interactions in shear and electrical fields. J Colloid Interface Sci 33:67-83. doi: 10.1016/0021-9797(70)90073-1

Utada AS, Lorenceau E, Link DR, et al (2005) Monodisperse Double Emulsions Generated from a Microcapillary Device. Science 308:537-541. doi: 10.1126/science.1109164

Wu Z, Gao Y, Li D (2009) Electrophoretic motion of ideally polarizable particles in a microchannel. Electrophoresis 30:773-781. doi: 10.1002/elps.200800381

Wu Z, Li D (2009) Induced-charge electrophoretic motion of ideally polarizable particles. Electrochim Acta 54:3960-3967. doi: 10.1016/j.electacta.2009.02.016

Wu Z, Li D (2008a) Micromixing using induced-charge electrokinetic flow. Electrochim Acta 53:5827-5835. doi: 10.1016/j.electacta.2008.03.039

Wu Z, Li D (2008b) Mixing and flow regulating by induced-charge electrokinetic flow in a microchannel with a pair of conducting triangle hurdles. Microfluid Nanofluidics 5:65-76. doi: 10.1007/s10404-007-0227-7

Yariv E (2005) Induced-charge electrophoresis of nonspherical particles. Phys Fluids 17:051702. doi: $10.1063 / 1.1900823$

Yossifon G, Frankel I, Miloh T (2007) Symmetry breaking in induced-charge electro-osmosis over polarizable spheroids. Phys Fluids 19:068105. doi: 10.1063/1.2746847

Zarzar LD, Sresht V, Sletten EM, et al (2015) Dynamically reconfigurable complex emulsions via tunable interfacial tensions. Nature 518:520-524. doi: 10.1038/nature14168

Zhang F, Daghighi Y, Li D (2011) Control of flow rate and concentration in microchannel branches by induced-charge electrokinetic flow. J Colloid Interface Sci 364:588-593. doi: 10.1016/j.jcis.2011.08.070

Zhang F, Li D (2014) A novel particle separation method based on induced-charge electroosmotic flow and polarizability of dielectric particles. Electrophoresis 35:2922-2929. doi: 10.1002/elps.201400194 
Table 1. Parameter values used in the simulations

Dielectric constant of the vacuum $\varepsilon_{0}(\mathrm{C} / \mathrm{Vm})$

$8.854 \times 10^{-12}$

Dielectric constant of the aqueous solution $\varepsilon$

80

Viscosity of the aqueous solution $\mu(\mathrm{kg} / \mathrm{ms})$

$0.9 \times 10^{-3}$

Density of the aqueous solution $\rho\left(\mathrm{kg} / \mathrm{m}^{3}\right)$

998

Zeta potential on positively charged surface of the Janus

60

droplet $\zeta_{p}(m V)$

Zeta potential on negatively charged surface of the Janus

$-40$

droplet $\zeta_{n}(m V)$

Droplet diameter $d(\mu m)$

200

Length of the simulation domain $L(\mu m)$

4000

Width of the simulation domain $W(\mu m)$

4000 


\section{Figure Legends}

Figure 1. Schematic of a sessile Janus droplet which is anchored on a solid surface in an aqueous solution.

Figure 2. Schematic diagram of the experimental system for studying electroosmotic flow field around a Janus droplet.

Figure 3. Schematic of the simulation domain for studying the fluid flow around a Janus droplet.

$$
\mathrm{L}=\mathrm{W}=20 \mathrm{~d} \text {. }
$$

Figure 4. Flow field around a Janus droplet under the electrical field of $40 \mathrm{~V} / \mathrm{cm}$. (a) numerical result with the red section carries negative charges and the blue section carries positive charges $\left(\zeta_{n}=-40 \mathrm{mV}, \zeta_{p}=60 \mathrm{mV}, \mathrm{d}=200 \mu \mathrm{m}\right)$; (b) experimental observation of a sessile Janus droplet partially covered with positively charged aluminum oxide nanoparticles under the same electric field (the diameter of the droplet $\mathrm{d}=170 \mu \mathrm{m})$.

Figure 5. Vortices around a Janus droplet under different applied electrical fields as predicted by numerical simulation. The red hemisphere carries negative surface charges and the blue hemisphere carries positive surface charges $\left(\zeta_{n}=-40 \mathrm{mV}, \zeta_{p}=60 \mathrm{mV}\right.$, $\mathrm{d}=200 \mathrm{um}$ ). (a) $\mathrm{E}=40 \mathrm{~V} / \mathrm{cm}$; (b) $\mathrm{E}=50 \mathrm{~V} / \mathrm{cm}$; (c) $\mathrm{E}=60 \mathrm{~V} / \mathrm{cm}$. (The direction of red arrows represents that of the fluid flow, and the length of red arrows is proportional to logarithmic flow velocity of the fluid.)

Figure 6. Experimentally observed vortices around the Janus droplet with the diameter of $170 \mu \mathrm{m}$ under different applied electric fields. (a) $\mathrm{E}=40 \mathrm{~V} / \mathrm{cm}$; (b) $\mathrm{E}=50 \mathrm{~V} / \mathrm{cm}$; (c) $\mathrm{E}=60 \mathrm{~V} / \mathrm{cm}$.

Figure 7. Vortices around Janus droplets with different surface areas covered by positive surface charges under electrical field $60 \mathrm{~V} / \mathrm{cm}$. The red section carries negative surface charges and the blue section carries positive surface charges $\left(\zeta_{n}=-40 m V, \zeta_{p}=\right.$ $60 \mathrm{mV}$, droplet diameter $\mathrm{d}=200 \mathrm{um})$. The surface coverage is indicated by the angle $\theta$. (a) $\theta=110^{\circ}$; (b) $\theta=60^{\circ}$. 
Figure 8. Experimentally observed vortices around Janus droplets with different surface coverages by the positively charged nanoparticles under electrical field $60 \mathrm{~V} / \mathrm{cm}$. The surface coverage is indicated by the angle $\theta$ as shown in Figure 7 . (a) $\theta \approx 110^{\circ}$, droplet diameter $\mathrm{d}=190 \mu \mathrm{m}$; (b) $\theta \approx 60^{\circ}$, droplet diameter $\mathrm{d}=170 \mu \mathrm{m}$.

Figure 9. The variation of the surface coverage of the Janus droplet and vortices around it with different strengths of externally applied electrical fields (droplet diameter $\mathrm{d}=215 \mu \mathrm{m}$ ). (a) $\mathrm{E}=40 \mathrm{~V} / \mathrm{cm}$; (b) $\mathrm{E}=60 \mathrm{~V} / \mathrm{cm}$. 


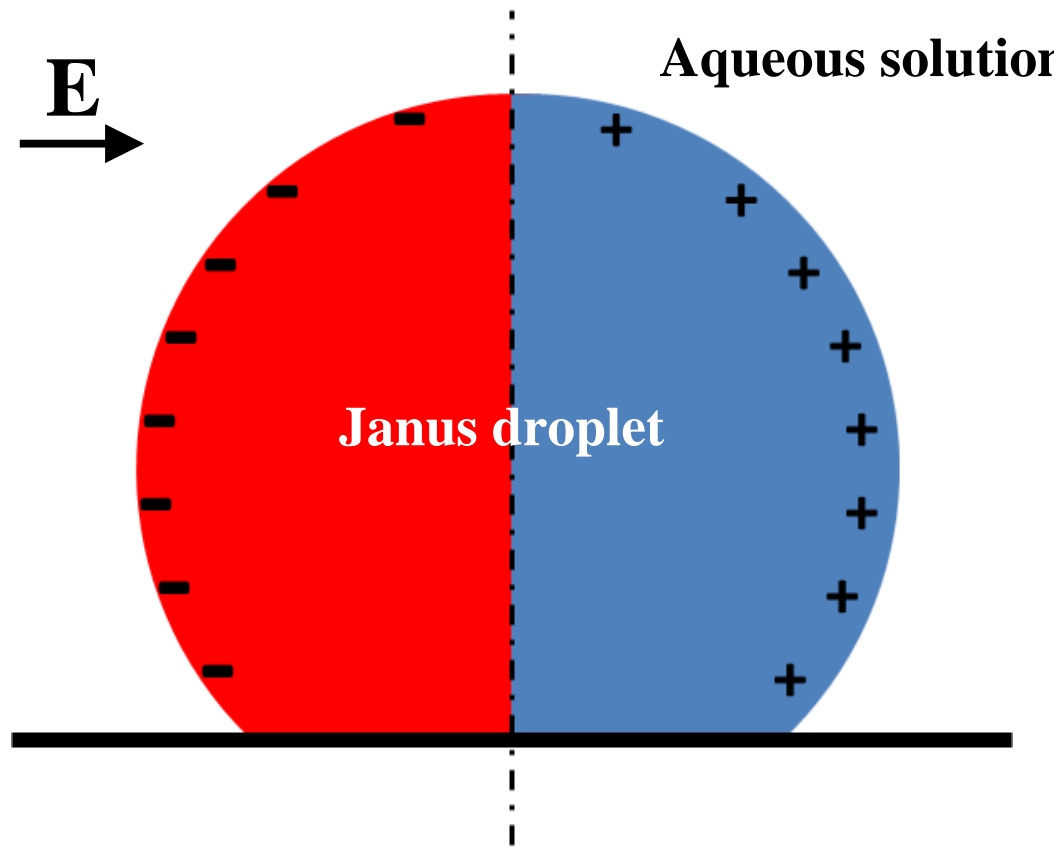

Figure 1 


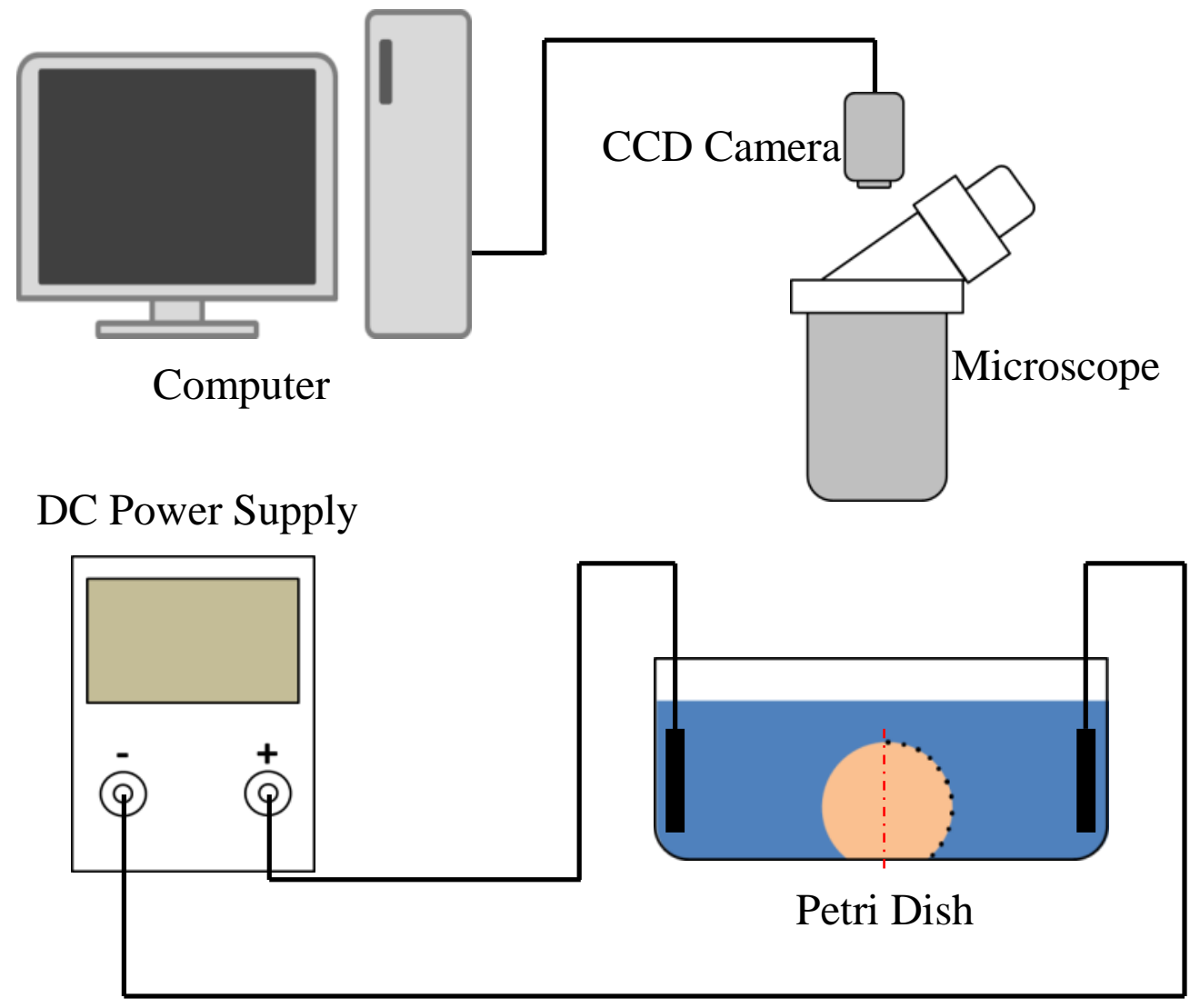

Figure 2 


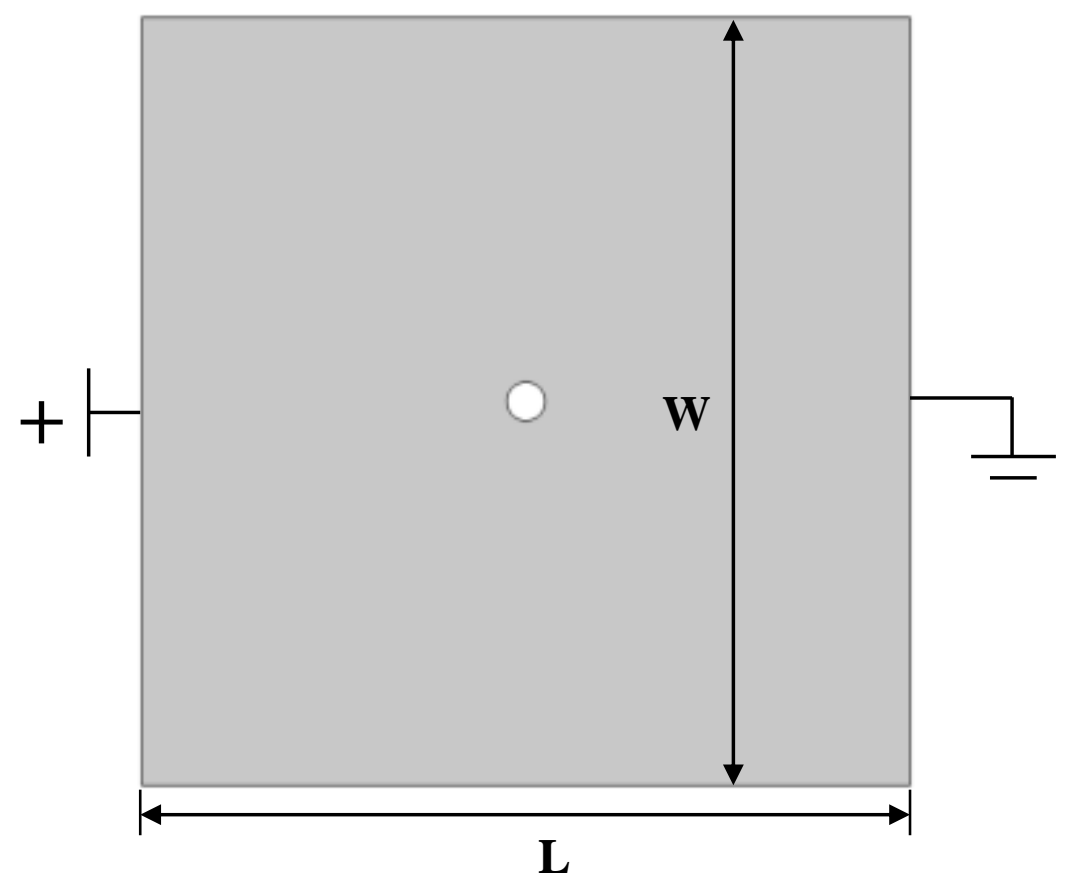

Figure 3 


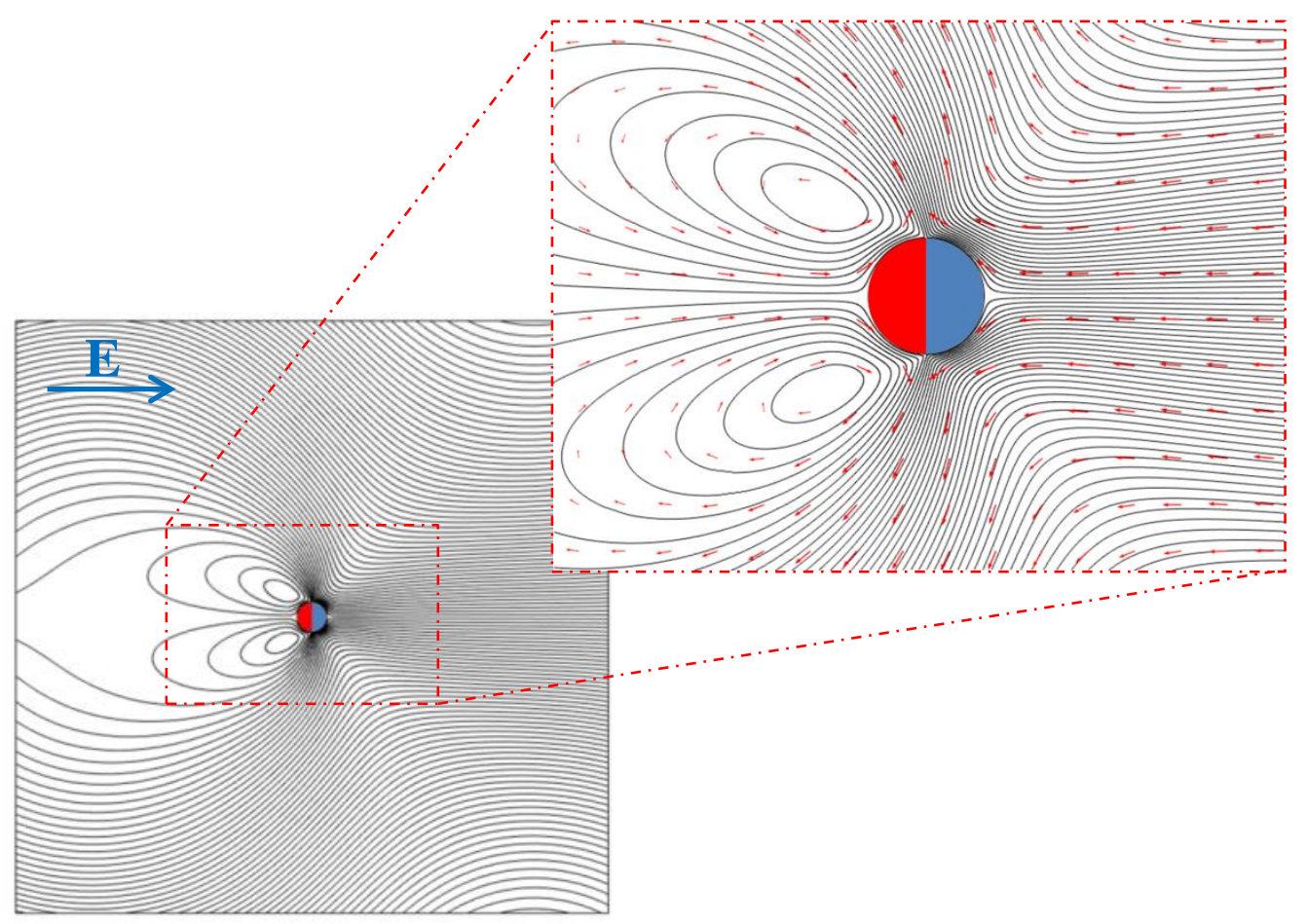

(a)

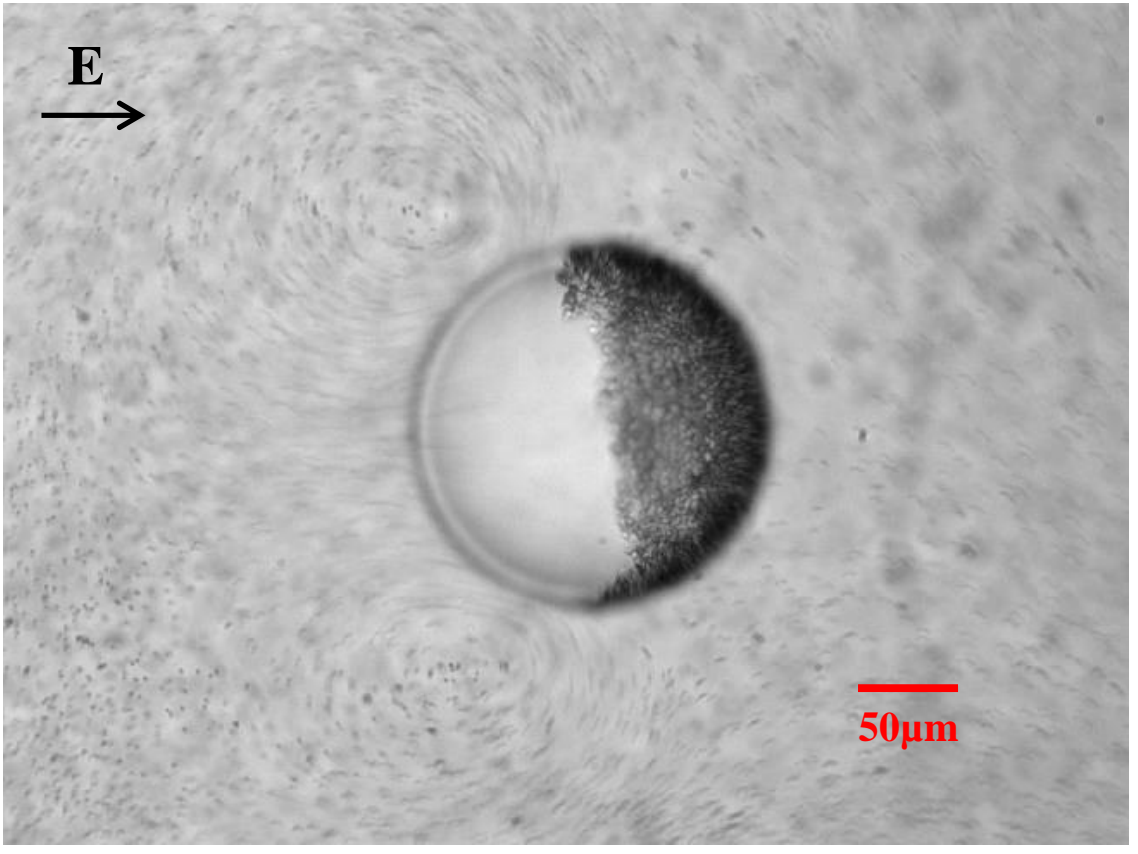

(b)

Figure 4 


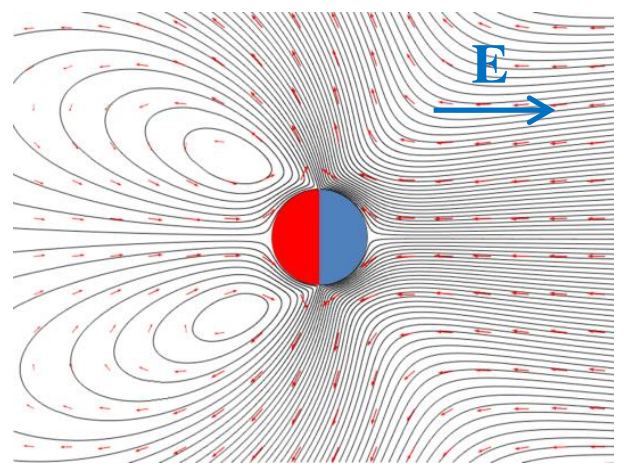

(a)

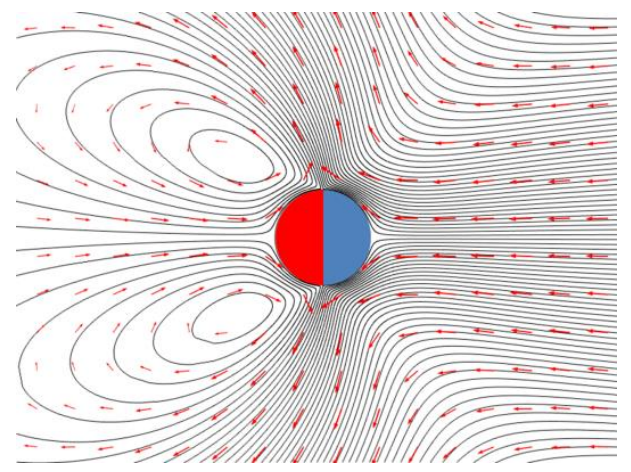

(b)

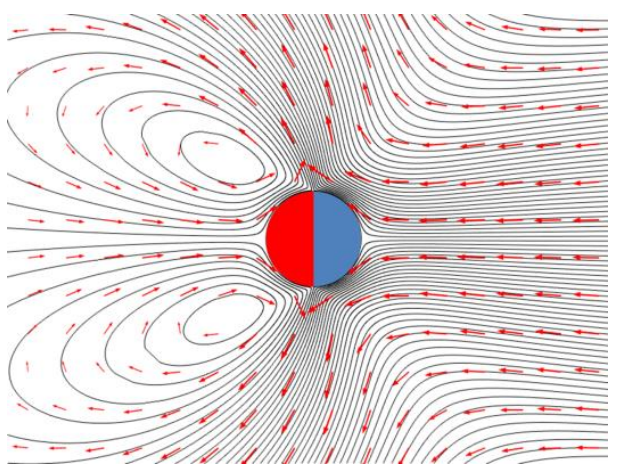

(c)

Figure 5 


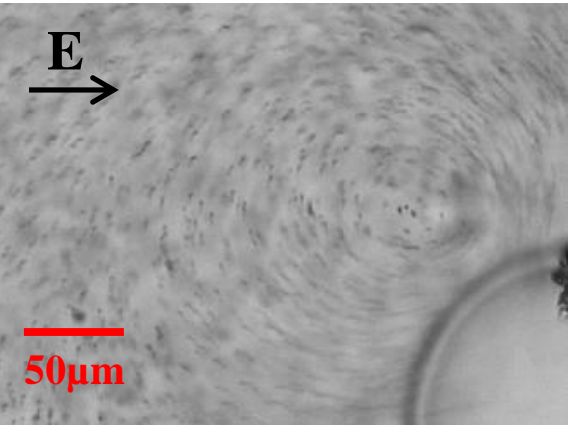

(a)

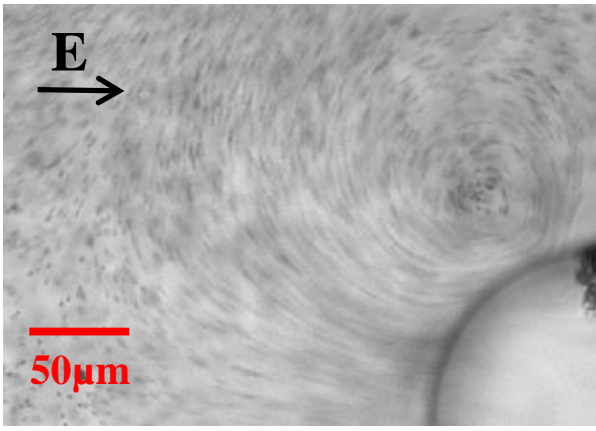

(b)

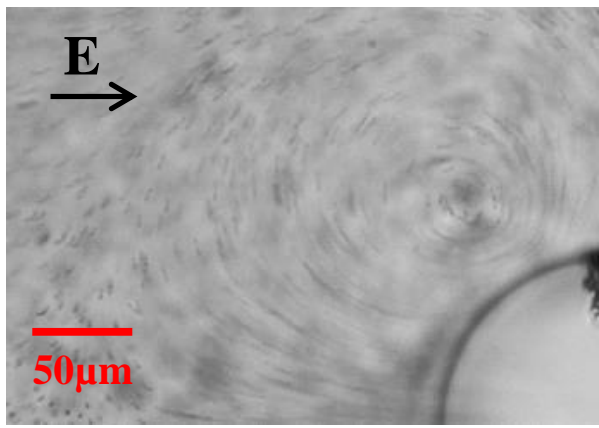

(c)

Figure 6 


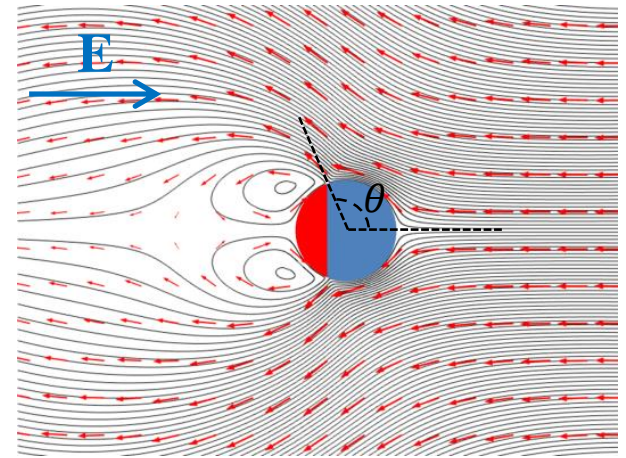

(a)

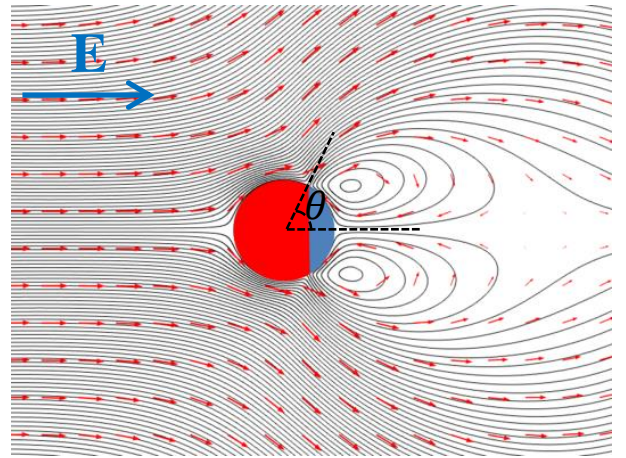

(b)

Figure 7 


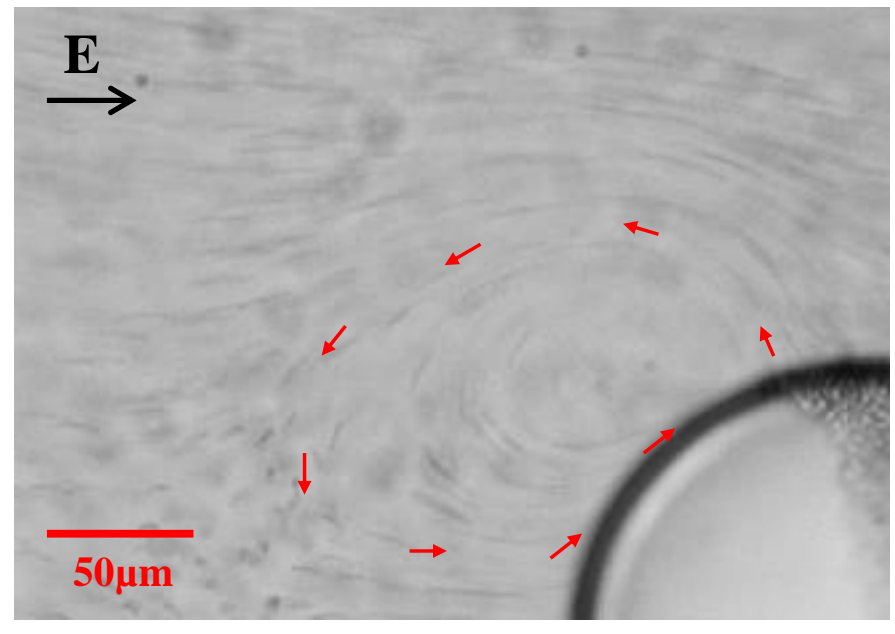

(a)

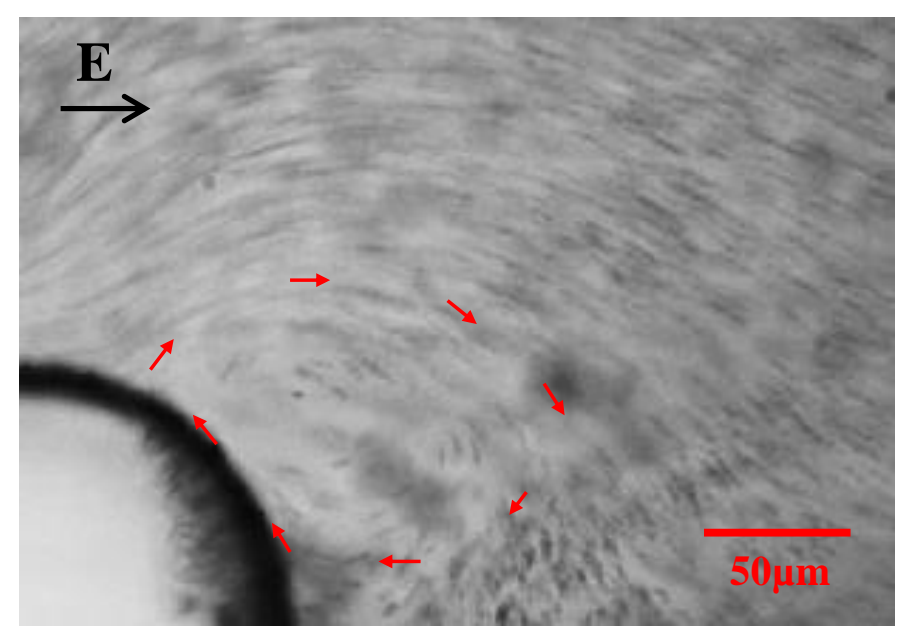

(b)

Figure 8 


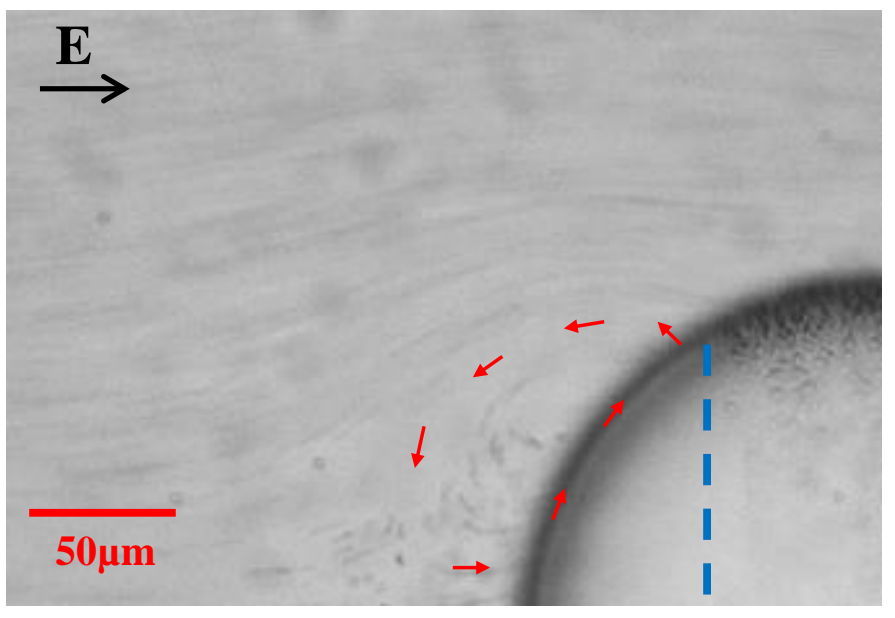

(a)

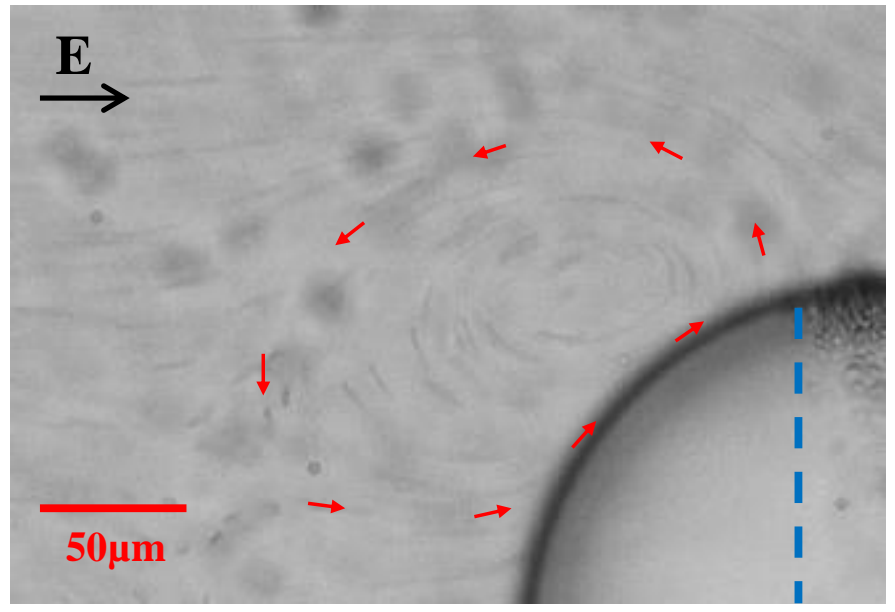

(b)

Figure 9 